\title{
Therapeutic Diet: What We Need to Consider!
}

\author{
Amrita Ghosh
}

\begin{abstract}
Therapeutic diet is needed so that nutritional factors may not affect individuals in diseases during convalescence and rehabilitation. Different research groups have observed a correlation between diseases and diets with diagnosis, prognosis, clinical outcomes and complications. In spite of considerable progress in healthcare, there is scarce evidence-based research excellence on community-level compliance on dietary advice. We need to change our mindset to find user-friendly dietary guidelines for the improvement in clinical approach and treatment in patients with diverse diseases in daily medical practice.
\end{abstract}

Keywords: Diet, Disease, Nutrition.

How to cite this article: Ghosh A. Therapeutic Diet: What We Need to Consider! Bengal Physician Journal 2018;5(2):25-27.

Source of support: Nil

Conflict of interest: None

\section{PROLOGUE}

Human body expects a balanced diet for normal functioning in health and disease. In compromised health settings there may be altered need of the diet pattern, both in its quality as well as in quantity. Qualified dieticians and knowledgeable health care providers are not easily available at handsbreadth in India to cater to the specialized need for specific illnesses. The therapeutic diet is routinely prescribed by attending doctors and planned by dieticians in the hospital practices which is difficult to get in domiciliary settings. Depending on the requirement of various macro- and micro-nutrients and texture therapeutic diet are transformed considering the caloric requirements. There are numerous explanations for the imbalance in appetite viz. anorexia, weakness, loneliness, self-pity and many other confounding factors including cooking practices, religious beliefs, traditions and customs of food habits; above all personal liking and disliking are of paramount importance. We have to be empathetic and compassionate to respect the illness behavior of the individual and convince them to comply with the prescribed unacceptable diet. ${ }^{1-3}$

Demonstrator

Department of Biochemistry, Medical College, Kolkata, West Bengal, India

Corresponding Author: Amrita Ghosh, Demonstrator, Department of Biochemistry, Medical College, Kolkata, West Bengal, India, e-mail: amritaghosh1973@yahoo.com

\section{Benefits of Therapeutic Diet}

The prime and indispensable role of therapeutic diet is to preserve the predisease health status and taming altogether clinical or apparent and subclinical or impending nutritional deficiencies. As per precise indispensable requirements, the diet is planned based on existing scientific knowledge and technologies to conserve health including food constituents to which individual may be allergic and/or atopic. Additional functions of therapeutic diets are to respect the compromised organs responsible for metabolism and excretion. Also by fine-tuning of the composition of normal diet helps homeostasis to adjust metabolize, nutrients and excrete harmful substances. ${ }^{4}$

\section{Adaptations to Therapeutic Diet}

There are ranges of footsteps of the transformation of regular as well as normal diet to the tailor-made diet for different morbidities. Firstly, we keep in the audit of energy requirements based on age, gender, and other related factors. Non-starch polysaccharides, i.e., roughages comprehensively becomes important constituent along with specific nutrients considering the history of intolerance to a specific type of food. Further, depending on the pathophysiology of the oropharyngeal anatomical region and esophagus, the composition and preparation of the diet have to be tailored to suit to solid, semisolid, soft, semiliquid or liquid diet for certain periods of the spells of illnesses. To revert to normal diet and/or normal life again we have to take again the same steps back to the regular dietary practices of the individual. ${ }^{5,6}$ Extraordinary attention needed to make all therapeutic diet easily digestible with due respect to a variety of options, choices, and exchanges to modify based on the requirement of medical conditions as an adjunct to the medical interventions. ${ }^{7,8}$

\section{Diet in Specific Conditions Usually Encountered in Healthcare}

\section{Adult Under-nutrition}

Under-nutrition takes place in adults in chronic health conditions with and without dearth of sufficient healthy and balanced diet. In this situation, we need (a) healthier food choices with high energy and high protein content, (b) initially light properly cooked food, (c) sufficient fluid intake, (d) micronutrient supplementation particularly vitamin A and E, iron and folic acid, zinc, calcium (e) include fresh pulpy and juicy fruits and green leafy vegetables, (g) meat, eggs, fish, kidney and liver, fish liver 
oils (for non-vegetarian), second generation milk products, soybean (for vegetarian), (h) whole grains to provide adequate roughages. ${ }^{9}$ To formulate this diet the calorie requirement of a person needs to be assessed depending on age, gender, and other morphological parameters and eucaloric diet have to be provided. ${ }^{10}$

High protein diet is usually recommended as protein deficiency go with an adult undernutrition. Protein-rich food added to carbohydrate-rich contents of diet that usually trigger satisfaction and fulfillment. ${ }^{11}$

\section{Low Sodium and/or Low Salt Diet}

Low sodium diet is recommended in generalized edema, hypertension, heart failure and in other medical conditions. Daily intake of sodium is usually restricted to a maximum of 1500 to $2300 \mathrm{mgs}$ in these situations with assessments and reassessments at intervals. ${ }^{12}$

\section{Low Protein Diet}

In a compromised health situation like liver and kidney diseases and with protein and other metabolic disorders consumption of protein usually restricted to 1 to 1.5 grams $/ \mathrm{kg}$ of body weight with evaluations at intervals. ${ }^{13}$

\section{Bland Diet}

In gastrointestinal diseases with epigastric discomfort, nausea, vomiting, acute diarrhoeal diseases, peptic ulcer diseases, and other conditions bland diet is usually suggested which is soft in texture, low in fiber with mild seasoning. Though named as bland diet, taste and calorie content should never be compromised. ${ }^{14}$

\section{Low Residue Diet}

In postoperative cases of recent bowel surgery viz. ileostomy, colostomy, and bowel resections, or even preparing for bowel surgeries, Crohn's or diverticular disease, and other related conditions this low fiber diet helps. The researchers feel that this temporary intake plan may reduce stool amount in large intestine with the assumption for rest to the bowel and dietary fibers should not be more than 10 to 15 grams per day. ${ }^{15}$

\section{Diabetes Mellitus}

The nutritional goals for diabetes management are to prevent microvascular and macrovascular complications and entails participatory learning of the patients concerning self-help lifestyle modifications including dietary options, choices of portions and exchanges in the planning of the diet. Dual downstream complications of dysglycaemia and dyslipidemia are managed with the maintenance of overall nutritional status as per age and gender with culture-specific religion sensitive dietary advice. Exchange and portion list needed to respect ethnogeographical variations of availability of groups of macronutrients having an equivalent amount of carbohydrate, protein, fat, and calories that can be easily exchanged with another in the list. Health care providers have to orient their mindset to ask socio-economic condition of the diabetics so that as they can follow the dietary advice for lifelong and can 'live with the disease' with minimum complications. ${ }^{16-19}$

\section{Wilson Disease}

In Wilson's disease dietary restriction per se is not enough to control copper accumulation in the eyes, brain, kidneys, and liver, with local impediments in specific organs including cirrhosis of the liver. Key interventions are pharmacotherapy to help excrete excess copper in urine and dietary zinc supplementation to reduce absorption of copper from the gut. Researchers opined that with optimum medication adherence, restriction of copper-rich food groups (except shellfish and liver) may not reduce complications. ${ }^{20,21}$

\section{Celiac Disease}

In celiac disease (CD) with a permanent gluten intolerance, patients must adhere to a lifelong avoidance of gluten-containing foods and foods which are naturally gluten-free but at risk of contamination. This dietary approach is all inclusive to avoid exacerbation of symptoms and to reduce short-term and long-term complications. Firstly, excluding gluten limits food choices and exchanges with unbalanced intake of macro and micronutrients which may lead to clinical or subclinical deficiencies. We need a patient-tailored dietetic protocol with strict adherence to the qualitative and quantitative composition of diets based on rich information on gluten-containing foods. Secondly, gluten free diet may not result in complete remission of mucosal damage or the resolution of symptoms and long trouble-free life. Thirdly, In comorbidity of irritable bowel syndrome (IBS) with celiac disease with comparable symptoms, a new therapeutic approach suggested avoidance of both gluten/wheat and fermentable oligosaccharides, disaccharides, monosaccharides, and polyols-shortchain carbohydrates and sugar alcohol (FODMAPs). ${ }^{22}$

\section{Standard Office Procedure Instead of 'Lip' Services}

The healthcare providers need to change themselves first to break the glass-box of fixed ideas and accept their limited knowledge about art and science of diet and nutrition based on limited translational researches on nutrition. Then only we can make sure that all health care professionals are to be consulted with respect to the diet plan and implementation in any morbidity. For this we need capacity building on diet and nutrition in undergraduate and postgraduate medical courses with the significance of therapeutic diet; special attention needed for family medicine specialty which is striving to champion primary care at grassroots. ${ }^{23,24}$ Further, our 
national policy should have been oriented to make people aware of the importance of foods for the comprehensive health care from womb to tomb in preventive, promotive and curative health care services. ${ }^{25}$

We have to start from likes-dislikes, family practice, religious beliefs and other constraints by identification of needs of the patients and extensive exchange of opinion from the community to reinforce the importance of diet as an important component of healing. This will help implementation challenges in method and time of meal plan with the patient and the patient's family, encourage them to depend on themselves to eat, provide adaptive equipment, right atmosphere. Clinical practice guideline needed with a comprehensive dedicated diet based on age, gender, morbidity, resource allocation, dedicated interventions updated every day from the care-seekers to revert them back with tailor-made advice. ${ }^{26}$ We need to exploit our health care delivery system for wider ramifications of our knowledge to the rural and urban poor people. ${ }^{27}$ In spite of important development in healthcare, the quality and quantity of translational research on diet nutrition are low on short, medium and long-term consequences of therapeutic diets. To sum up, we have to shift from conventional 'ready-made diet chart approach' to 'tailor-made approach' with a human face to think empathetically-When I am the patient, what would be the best diet for me! $!^{28,29}$

\section{REFERENCES}

1. Gopalan C, Rama Sastri BV and Balasubramanian SC, Nutritive value of Indian foods, National Institute of Nutrition, ICMR, Hyderabad, India, 2004.

2. Therapeutic Diet. J. Clin Nutri Diet. [online] [cited on 05.10.2018] Retrieved from: http://www.imedpub.com/scholarly/therapeutic-diet-journals-articles-ppts-list.php.

3. Rustagi N, Pal R, Dudeja P. Good Cooking Practices in Kitchen in Eds. Dudeja P, Singh A, Kaur S. Food Safety Implementation: from Farm to Fork. CBS Publishers and Distributors Pvt. Ltd.1st ed. New Delhi. 2016; Chapter 20, pp 181-190.

4. The Importance of Therapeutic Diets. [online] [cited on 05.10.2018] Retrieved from: https://www.pedagogyeducation. com/Main-Campus/Student-Union/Campus-Blog/September-2013/The-Importance-of-Therapeutic-Diets.aspx.

5. Therapeutic diets. [online] [cited on 01.10.2018] Retrieved from: https://www.laps.in/Therapeutic.pdf.

6. Fad Food Diets vs Custom Therapeutic Diets. [online] [cited on 01.10.2018] Retrieved from: https://www.empowerhealth. ca/fad-food-vs-therapeutic-diet/.

7. Types of therapeutic diets. [online] [cited on 01.10.2018] Retrieved from:http://www.cdss.ca.gov/agedblinddisabled/ res/VPTC2/9\%20Food\%20Nutrition\%20and\%20Preparation/ Types_of_Therapeutic_Diets.pdf

8. Types of therapeutic diet. [online] [cited on 01.10.2018] Retrieved from:https://www.livestrong.com/article/320563types-of-therapeutic-diets/.
9. WHO/Nutrition for older persons. [online] [cited on 01.10.2018] Retrieved from: http://www.who.int/nutrition/ topics/ageing/en/index1.html.

10. 17 Best Foods for Dieters. [online] [cited on 01.10.2018] Retrieved from: https://www.webmd.com/diet/obesity/ features/17-best-foods-for-dieters\#4.

11. A High-Protein Diet Plan to Lose Weight and Improve Health. [online] [cited on 28.08.2018] Retrieved from: https://www. healthline.com/nutrition/high-protein-diet-plan.

12. Sodium-Controlled Diet. [online] [cited on 01.10.2018] Retrieved from: https://my.clevelandclinic.org/health/ articles/15426-sodium-controlled-diet.

13. A Complete Guide to a Low-Protein Diet. [online] [cited on 01.10.2018] Retrieved from: https://www.healthline.com/ nutrition/low-protein-diet.

14. Bland Diet:What to Eat and What to Avoid. [online] [cited on 01.10.2018] Retrieved from: https://www.healthline.com/ health/food-nutrition/bland-diet.

15. Should You Try a Low-Residue Diet? [online] [cited on 01.10.2018] Retrieved from: https://www.webmd.com/ibdcrohns-disease/low-residue-diet-foods\#1.

16. Garg R, Chawla SPS, Pal R, Ghosh A. Impact of Health Education on Knowledge, Attitude, Practices and Glycemic Control in Type 2 Diabetes Mellitus. J Fam Med Primary Care 2018. [In Press].

17. Ghosh A, Pal R. Glycemic control in Hypothyroidism and Type 2 Diabetes Mellitus. Published in the proceedings of WONCA 15th World Rural Health Conference WRHC 2018, New Delhi, April 2018.

18. Singh M, Pal R, Ranjan R, Sarker G, Bharati DR, Pal S. Diabetes and Dementia: Myth and reality. J Krishna Inst Med Sci Uni 2017;6(4):12-17.

19. Pal R, Pal S, Barua, Ghosh MK. Health Education intervention on Diabetes in Sikkim. Indian J Endocr Metab 2010;14(1): 3-7.

20. Low Copper Diet for Wilson's Disease. [online] [cited on 01.10.2018] Retrieved from: https://www.gicare.com/gihealth-resources/copper-restriction/

21. Dietary Copper Restriction in Wilson's disease. Russel K, Gillanders Lyn K, Plank L D, Eur J Clin Nutri 2018;72:326-331.

22. Taus M, Mignini EV, Fumelli D, Busni D, Nicolai G, Carletti C, et al. Celiac Disease: Gluten Free Diet and...What Else? Open J Gastroenterol 2016;6:157-170.

23. Pal R, Kumar R, Vidyasagar, Rustagi N, Mukherjee B, Sarbapalli D. Future direction of Family Medicine training in India. J Family Med Prim Care 2014;3(4):295-299.

24. Pal R, Kumar R, Pal S, Vidyasagar, Mukherjee B, Sarbapalli D. Medical Education: The Hot Seat. J Family Med Prim Care 2016;5: 20-23.

25. Sharma S, Singh M, Pal R, Ranjan R, Pal S, Ghosh A. National Health Policy 2017: Can it lead to achievement of sustainable development goals? Al Ameen J Med Sci 2018;11(1):4-11.

26. Pal R. Everything is learning. Educ Health (Abingdon) 2017; 29(3):271-272

27. Saxena V, Kumar P, Kumari R, Nath B, Pal R. Availability of Village Health and Nutrition Day services in Uttarakhand, India. J Fam Med Primary Care 2015;4:251-256

28. Pal R, Rustagi N. Food safety: Who's who. J Med Nutr Nutraceut 2015;4:27-31.

29. Pal R. Mohanta PK, Rustagi N, Sarbapalli D, Ghosh A, Sarker G. Knowledge is power if you hone it. Int J Green Pharm 2015;9(2):86-89. 